\title{
Spatially Resolved Measurement of the Stress Tensor in Thin Membranes Using Bending Waves
}

\author{
Reimar Waitz, ${ }^{*}$ Carolin Lutz, ${ }^{\dagger}$ Stephan Nößner, ${ }^{\ddagger}$ Michael Hertkorn, ${ }^{\S}$ and Elke Scheer ${ }^{\|}$ \\ Department of Physics, University Konstanz, 78457 Konstanz, Germany
}

\begin{abstract}
The mode shape of bending waves in thin silicon and silicon carbide membranes is measured as a function of space and time, using a phase shift interferometer with stroboscopic light. The mode shapes hold information about all the relevant mechanical parameters of the samples, including the spatial distribution of static prestress. We present a simple algorithm to obtain a map of the lateral tensor components of the prestress, with a spatial resolution much better than the wavelength of the bending waves. The method is not limited to measuring the stress of bending waves. It is applicable in almost any situation, where the fields determining the state of the system can be measured as a function of space and time.
\end{abstract}

\section{INTRODUCTION}

Mechanical waves are a powerful tool to study material properties over a broad range of length scales. The applications range from seismic-wave methods to studying the structure of the Earth [1], SONAR for underwater location and navigation [2], ultrasonic testing of materials in engineering $[3,4]$ and medical ultrasonography [3], down to methods applicable to micro- and nanostructures like acoustic microscopy [3], Brillouin scattering [5], and picosecond pump-probe spectroscopy [6,7]. For all these methods, the length scale of the inhomogeneities of the material properties is supposed to be larger than the wavelength.

In a previous work [8], we show how to extract the dispersion relation of bending waves of prestressed thin plates from measured mode shapes. Stress and bending stiffness of the membrane are determined as fit parameters using a theoretical model of the dispersion relation. Since the existence of a well-defined dispersion relation requires a homogeneous system, this method has the same shortcomings as the methods listed above. Here, we present an approach using full knowledge of the wave function to determine the mechanical properties of the sample. We measure the shape of a bending-wave mode in direct space and compare it to the equation of motion. The parameters found in the equation are the components of the stress tensor divided by the density of the membrane $\sigma_{i j} / \rho$, as

\footnotetext{
*Present address: Rational AG, 86889 Landsberg am Lech, Germany.

${ }^{\dagger}$ Present address: National Institute for Materials Science and Waseda University, 121 Sengen, Tsukuba Ibaraki, 3050047 Japan.

${ }^{\ddagger}$ Present address: Robert Bosch GmbH, 71701 Schwieberdingen, Germany.

${ }^{\S}$ Present address: ZF Friedrichshafen AG, 88046 Friedrichshafen, Germany.

"elke.scheer@uni konstanz.de
}

well as a constant proportional to the bending stiffness. With our simple algorithm, these parameters are obtained as a function of space using a linear fit. Since the full mode shape is available and we are not limited to far-field information, the wavelength of the bending waves is no limit for the spatial resolution. For the measurement of the stress-tensor components of semiconductors, all optical methods can be applied with spatial resolution in the order of a few micrometers [9,10] to roughly $100 \mu \mathrm{m}$ [11], depending on the respective methods. However, these methods make use of particular optical properties and cannot straightforwardly be generalized to insulators or metals.

\section{EXPERIMENT}

For qualifying the methods, two different types of membranes are studied: single-crystal silicon membranes as well as silicon-carbide membranes. Both types are a few hundred nanometers thick and have a lateral size of roughly $500 \mu \mathrm{m}$. They are fabricated from coated silicon wafers using a wet-etching process [8] adapted from Ref. [[12]]. The wafers used are standard silicon-on-insulator wafers and silicon-carbide-coated silicon wafers [13].

The flatness of the silicon membranes is better than $1 \%$ of the lateral size. A small static buckling is caused by the formation of native oxide leading to a compressive stress $[8,14,15]$. During the measurement, we apply a pressure difference of 50 mbar between the top and the bottom side of the membrane, so it is bent by $8 \mu \mathrm{m}$. As a consequence, there is an inhomogeneous and anisotropic tensile stress.

The silicon-carbide films and therefore the membranes have an average tensile stress of tens to hundreds of megapascals, so there is no need to use air pressure to stretch them. The tensile stress is highest at the bottom side of the membrane and lowest on the top. We use the radius of curvature of a curled free-membrane flake to estimate 
the stress gradient perpendicular to the membrane plane. When the top layer of the membrane is removed, the average stress increases. Therefore, we can design spatially resolved stress patterns using reactive ion etching with an $\mathrm{SF}_{6}-\mathrm{O}_{2}$ plasma using an etch mask defined by electronbeam lithography.

A sketch of the experimental setup used for measuring the bending waves of silicon membranes is shown in Fig. 1(a). The membrane samples consist of freestanding membrane films connected to a rectangular solid frame, that is glued to a piezoelectric ring. Applying an ac voltage, we induce thickness oscillations of the piezoelectric ring and therefore couple vibrations into the membrane. Using an imaging interferometer with a stroboscopic light source, we measure the surface profile $z(\vec{r}, t)$ of the oscillating membrane as a function of space and time. Two examples of $z(x, y)$ measured at fixed time for two different frequencies are given in Fig. 2. Using a Fourier series in time, the mode shape of frequency eigenmodes $\tilde{z}(\vec{r}, \omega)$ is calculated from the time-domain data $z(\vec{r}, t) . \omega$ stands for the angular frequency of the excitation or a higher harmonic of it. For measurements on silicon-carbide membranes, a similar excitation mechanism is used, but the whole sample is placed inside a vacuum cell. In this case, it is not possible and not necessary to apply a pressure difference between the top and the bottom side. The setup used for measurements in vacuum is shown in Fig. 1(b). More detail about the experimental procedure to measure the vibrational modes $\tilde{z}(\vec{r}, \omega)$ at several frequencies $\omega$ has been published in a previous paper [8]. Although the resolution of the amplitude is much better than $1 \mathrm{~nm}$, for the analysis here, we use amplitudes in the range of $1 \mathrm{~nm}$ to several $100 \mathrm{~nm}$. The upper limit of the useful amplitudes is given by instrumental limits (interference fringes get washed out at higher amplitudes) and by nonlinear effects of the mechanical system. The lower limit is given by the signal-to-noise ratio (SNR). Modes with poor SNR enhance the calculation effort but do not improve the resolution of the stress images. As we will show, the three

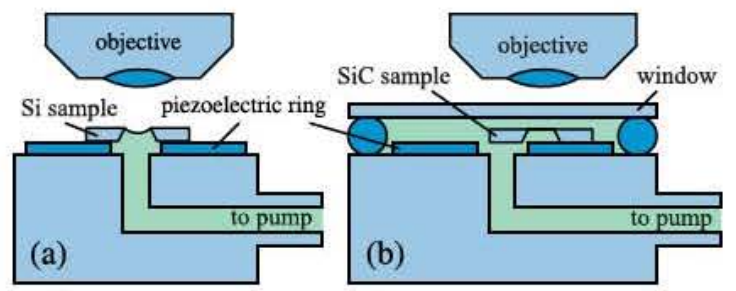

FIG. 1. Sketch of the experiment showing schematically the mounting of membrane samples in the optical profilometer. The sample is glued to a piezoelectric ring used to excite the vibrations. (a) For measurements on silicon membranes, we use a pump to apply a pressure difference between the upper and lower sides of the membrane. (b) Measurements on silicon carbide membranes are performed in a vacuum cell.
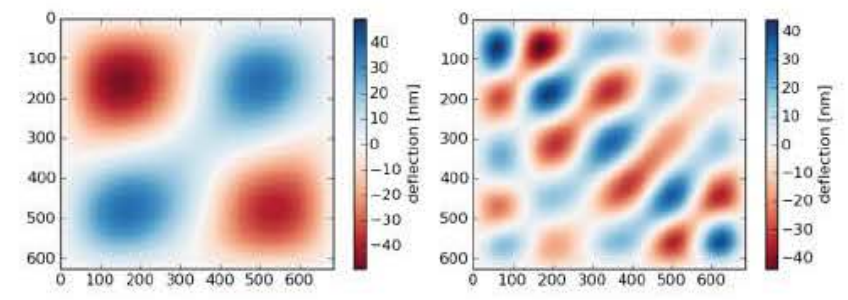

FIG. 2. Examples of amplitude profiles measured on a silicon carbide membrane with size $685 \times 625 \mu \mathrm{m}^{2}$, recorded at an excitation frequency of $f=393.1 \mathrm{kHz}$ (left) and $1.020 \mathrm{MHz}$ (right). These modes feature relatively high amplitude because they correspond to eigenmodes of the system [left, $(2,2)$ mode and right, $(5,5)$ mode, where $(n, m)$ denotes the number of extremes in $x$ and $y$ directions].

independent components of the stress tensor $\sigma_{i j}$ are unambiguously determined by the mode shapes $\tilde{z}(\vec{r}, \omega)$.

\section{EQUATION OF MOTION OF A THIN PLATE WITH INHOMOGENEOUS PRESTRESS}

As we have shown in an earlier work [8], the equation of motion for the deflection $z$ of a homogeneously prestressed thin plate is

$$
-\ddot{z}+\underbrace{\sum_{i, j \in\{x, y\}} \frac{\sigma_{i j}}{\rho} \frac{\partial^{2} z}{\partial_{i} \partial_{j}}}_{\text {prestressed membrane }}-\underbrace{\frac{D}{h \rho} \Delta^{2} z}_{\text {thin plate }}=0,
$$

with the density $\rho$ and the stress tensor $\sigma_{i j}$ both averaged over the thickness $h$ of the plate. The bending stiffness $D=E h^{3} /\left[12\left(1-\nu^{2}\right)\right]$ depends on Young's modulus $E$ and the Poisson ratio $\nu$. $\Delta$ denotes the Laplace operator. The first addend in Eq. (1) corresponds to the inertia of the system, the second one to the restoring force of a prestressed membrane, the third one to the bending force of a thin plate [16-19]. In the following, we show that Eq. (1) still holds for inhomogeneous stress.

The general form of the prestressed membrane term is known from the literature [17],

$$
\sum_{i, j \in\{x, y\}} \frac{1}{\rho}\left(\frac{\partial \sigma_{i j}}{\partial_{i}} \frac{\partial z}{\partial_{j}}+\sigma_{i j} \frac{\partial^{2} z}{\partial_{i} \partial_{j}}\right) .
$$

In our system, the phase velocity for longitudinal waves is much larger than the one of bending waves, because Young's modulus is large compared to the stress-tensor components. Therefore the lateral motion is considered to be always in equilibrium during the bending-wave oscillation. Using the condition for lateral equilibrium [17],

$$
\sum_{j} \frac{\partial}{\partial_{i}} \sigma_{i j}=0,
$$


the general term in Eq. (2) simplifies to the homogeneousstress term used in Eq. (1).

The equivalence of the equations for the homogeneous and the inhomogeneous case, however, is not self-evident. For example, in case of an inhomogeneous bending stiffness $D$, the equation of motion becomes much more complex, including several spatial derivatives of $D$. As a result, there are four additional addends to Eq. (1). In principle, the method described below would still be applicable using four more fit parameters. In practice, a fit with an excessive number of free parameters requires experimental data with unachievable precision and is therefore not viable. For that reason, we limit ourselves to systems for which $D$ is constant or negligible.

\section{FIT METHOD}

In this section, we present a method to calculate the spatially resolved mechanical constants of the system from measured mode shapes $z$. The knowledge of two quantities is required: First we need the field $z(\vec{r}, t)$ as a function of space $\vec{r}$ and time $t$. For oscillatory linear systems with an angular frequency $\omega$, it is advantageous to use the harmonic ansatz

$$
z_{\omega}(x, y, t)=\tilde{z}(x, y, \omega) e^{i \omega t}
$$

and to work with $\tilde{z}(\vec{r}, \omega)$ instead of $z_{\omega}(\vec{r}, t)$. In general, the following procedure works both in the time domain as well as in the frequency domain, but in the following we restrict ourselves to the frequency domain. A false-color image of the measured mode $\tilde{z}(\vec{r}, \omega)$ is shown in Fig. 3, highlighted by a blue frame. Since the method works for arbitrary $\omega$, we demonstrate it here for a higher order mode with many maxima and minima (blue and red areas).

The second requirement is an equation that can be written as

$$
0=\sum_{l} p_{l}(\vec{r}) \hat{X}_{l} z
$$

with operators $\hat{X}_{l}$, which are linearly independent and which do not depend on any unknown parameters. Usually some of the coefficients $p_{l}$ are well-known constants while others correspond to the material properties we are looking for. Let $\Omega_{\text {unknown }}$ be the set of indices $l$ of the unknown properties $p_{l}$, so our goal is to extract the values for the coefficients $p_{l}$ with $l \in \Omega_{\text {unknown }}$.

The equation of motion for prestressed thin plates [Eq. (1)] is equivalent to Eq. (4) using the parameters $p_{l}$ and the operators $\hat{X}_{l}$ shown in Table I. Using the harmonic ansatz in Eq. (3), we can replace $-\ddot{z}$ by $\omega^{2} z$. $\omega$ is known from the experiment, $h$ is measured separately by ellipsometry, and $\rho, E, \nu$ are taken from literature, so $D$ is a known constant. There are three unknown parameters $\left(\Omega_{\text {unknown }}=\{2,3,4\}\right)$ in Table I, corresponding to the

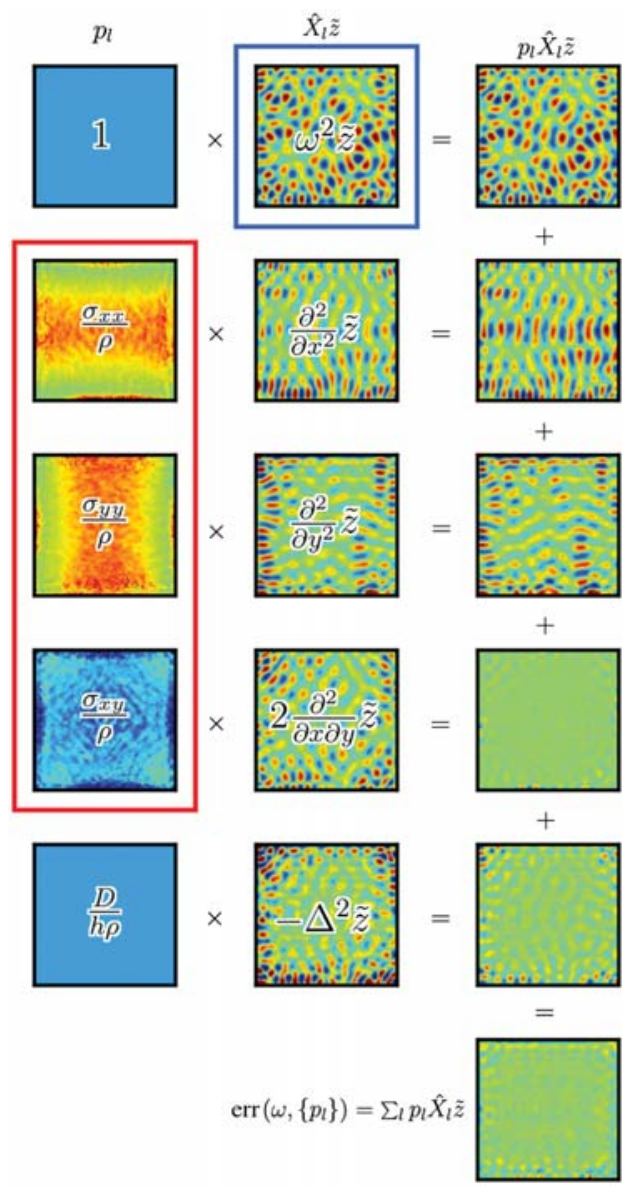

FIG. 3. Visualization of the fitting method showing all the addends in the equation of motion [Eqs. (1) and (4)] for a mode at one fixed frequency $\omega$. The sample shown here is a $340 \mathrm{~nm}$ thick silicon membrane with a lateral size of $714 \times 691 \mu \mathrm{m}^{2}$ bent by a pressure difference of 50 mbar. The measured mode shape is highlighted by a blue frame. In this case, it is a higher order mode with many maxima and minima. The goal is to obtain the stress tensor components highlighted by a red frame. The waveforms $\hat{X}_{l} \tilde{z}$ in the center column are calculated using numerical deriv atives on the measured mode shape. Each row corresponds to one addend with one value for the index $l$. The parameters $p_{l}$ are either trivial (1), known from the geometry $(D / h \rho)$, or they can be treated as free fit parameters $\left(\sigma_{i j} / \rho\right.$, red frame). In the right column, the product $p_{l} \hat{X}_{l} \tilde{z}$ is shown. The image at the bottom right is the sum $\sum_{l} p_{l} \hat{X}_{l} \tilde{z}$, which should be equal to zero according to the equation of motion [Eq. (4)]. This equality is used as a condition to find the free parameters (red frame). All the false color images use arbitrary units, with green corresponding to zero, blue to negative, and red to positive values. For the complex quantities $\hat{X}_{l} \tilde{z}_{\text {and }} p_{l} \hat{X}_{l} \tilde{z}$, the real part is shown.

components of the stress tensor. A graphical visualization of Eq. (4) is shown in Fig. 3. The panels in the first column show false-color images of the parameters $p_{l}(\vec{r})$. The parameters with $l \in \Omega_{\text {unknown }}$ (highlighted by a red frame), corresponding to the components of the stress tensor, are the quantities we are looking for. The procedure to calculate 
TABLE I. Inserting the coefficients $p_{l}$ and operators $\hat{X}_{l}$ from this table into the general equation of motion [Eq. (4)] leads to the equation for prestressed thin plates [Eq. (1)].

\begin{tabular}{|c|c|c|c|c|c|}
\hline$l$ & 1 & 2 & 3 & 4 & 5 \\
\hline$p_{l}$ & 1 & $\sigma_{x x} / \rho$ & $\sigma_{y y} / \rho$ & $\sigma_{x y} / \rho$ & $D / h \rho$ \\
\hline$\hat{X}_{l}$ & $-\left(\partial^{2} / \partial t^{2}\right)$ or $\omega^{2}$ & $\partial^{2} / \partial x^{2}$ & $\partial^{2} / \partial y^{2}$ & $2\left(\partial^{2} / \partial x \partial y\right)$ & $-\Delta^{2}$ \\
\hline
\end{tabular}

them from the experimental mode shapes is explained below.

Equation (4) is equivalent to

$$
\operatorname{err}\left(\omega,\left\{p_{l}(\vec{r})\right\}, \vec{r}\right)=0
$$

for all $\omega$, using the single-frequency error function,

$$
\operatorname{err}\left(\omega,\left\{p_{l}(\vec{r})\right\}, \vec{r}\right)=\sum_{l} p_{l}(\vec{r}) \hat{X}_{l} \tilde{z}(\vec{r}, \omega) .
$$

Equations (5) and (6) give one equation per mode. Therefore, at least as many modes as unknowns are needed, if all the equations are linearly independent. Even under ideal conditions with zero noise, linear independence is not necessarily the case. To account for this problem, at least one additional equation, meaning one additional mode, is necessary. More modes increase the SNR in the stresspattern images; see below. The sum Eq. (6) is illustrated in Fig. 3 for an arbitrary but fixed frequency $\omega$. Each row in the figure corresponds to one of the addends in the equation, defined by the product $p_{l}(\vec{r}) \hat{X}_{l} \tilde{z}(\vec{r}, \omega)$. On the left-hand side of the figure, there is a false-color image of the best-fit values of $p_{l}(\vec{r})$. How to calculate them from the data is explained below. $\hat{X}_{l} \tilde{z}(\vec{r}, \omega)$ can be calculated from the experimental data. $\hat{X}_{l} \tilde{z}(\vec{r}, \omega)$ is depicted in the center column. On the right-hand side of the figure, a false-color image of each addend, the product $p_{l} \hat{X}_{l} \tilde{z}$, is shown. $\operatorname{err}\left(\omega,\left\{p_{l}(\vec{r})\right\}, \vec{r}\right)$, defined by the whole sum, is shown in the bottom right panel. Since err is calculated from measured data with finite uncertainty, it is not exactly equal to zero, but it is much smaller than one addend on its own.

The total error function is defined as

$$
\operatorname{Err}\left(\left\{p_{l}(\vec{r})\right\}, \vec{r}\right)=\sum_{\omega} w(\omega)\left|\operatorname{err}\left(\omega,\left\{p_{l}(\vec{r})\right\}, \vec{r}\right)\right|^{2} \geq 0
$$

The sum runs over all the modes measured at several frequencies $\omega$. The choice of the weight function $w(\omega)>0$ is arbitrary; in our example we use

$$
w(\omega)=\frac{1}{\left\langle\left|\omega^{2} \tilde{z}(\omega)\right|^{2}\right\rangle},
$$

with the spatial average $\langle\cdot\rangle$, so the average contribution of the inertia term $\omega^{2} z$ is the same for all frequencies.
Other weighting functions can be chosen such that modes with high amplitude have more weight to improve the SNR. Equation (5) and therefore also the equation of motion [Eq. (4)] is equivalent to

$$
\operatorname{Err}\left(\left\{p_{l}(\vec{r})\right\}, \vec{r}\right)=0 .
$$

The equality only holds for exact solutions $\tilde{z}$ and the correct parameters $p_{l}$. When measured data with finite uncertainty are used instead, Err is always positive and it is minimal, when the unknown parameters $p_{l}$ with $l \in \Omega_{\text {unknown }}$ are close to their true values. The minimum is found using the condition

$$
\frac{\partial}{\partial p_{l}} \operatorname{Err}\left(\left\{p_{l}(\vec{r})\right\}, \vec{r}\right)=0, \quad l \in \Omega_{\text {unknown }},
$$

leading to a system of linear equations,

$$
\begin{aligned}
0 & =\sum_{k} A_{l k}(\vec{r}) p_{k}(\vec{r}), \quad l \in \Omega_{\text {unknown }}, \\
A_{l k}(\vec{r}) & =\sum_{\omega} w(\omega) \operatorname{Re}\left\{\left(\hat{X}_{l} \tilde{z}\right)^{*}\left(\hat{X}_{k} \tilde{z}\right)\right\},
\end{aligned}
$$

where $\mathrm{Re}$ is the real part and the asterisk represents the complex conjugate. First, $A_{l k}$ is calculated using the numerical derivatives [see Table I and the center column of Fig. 3] on the measured modes $\tilde{z}(\vec{r}, \omega)$, then the system of linear equations, Eq. (11), is solved to obtain the best-fit values of the parameters $p_{k}$ with $k \in \Omega_{\text {unknown }}$ shown in the red frame in Fig. 3. Since the method explained above and especially the numerical derivatives are sensitive to noise in the experimental data, filtering of $z$ might be necessary. We use a two-step filtering process. First, the data are smoothed by a Gaussian bandpass filter centered around the mean wave number of the given mode with a variance of twice the mean wave number. Since some of the modes are excited with a much smaller amplitude than others, there are large variations in the SNR. Therefore, as a second filtering step, modes with low SNR are removed from the data set. The mode selection is easily automated in an iterative process using the tools presented above. First, the method is applied to the whole data set to obtain some initial values for $p_{l}$, then the modes with high average $\operatorname{err}\left(\omega,\left\{p_{l}(\vec{r})\right\}, \vec{r}\right)$ are removed and the procedure is repeated.

\section{RESULTS}

We find the components of the stress tensor $\sigma_{i j}$ by multiplying the $p_{l}$ with the mass density of silicon [20] $\rho_{\mathrm{Si}}=2330 \mathrm{~kg} / \mathrm{m}^{3}$ or silicon carbide [21] $\rho_{\mathrm{SiC}}=$ $3210 \mathrm{~kg} / \mathrm{m}^{3}$. The experimental values $\sigma_{i j}$ on siliconmembranes bent by a pressure difference of 50 mbar are compared to finite-element simulations. The results are shown in Fig. 4. Details about the simulation have been published in an earlier paper [8]. For the diagonal 

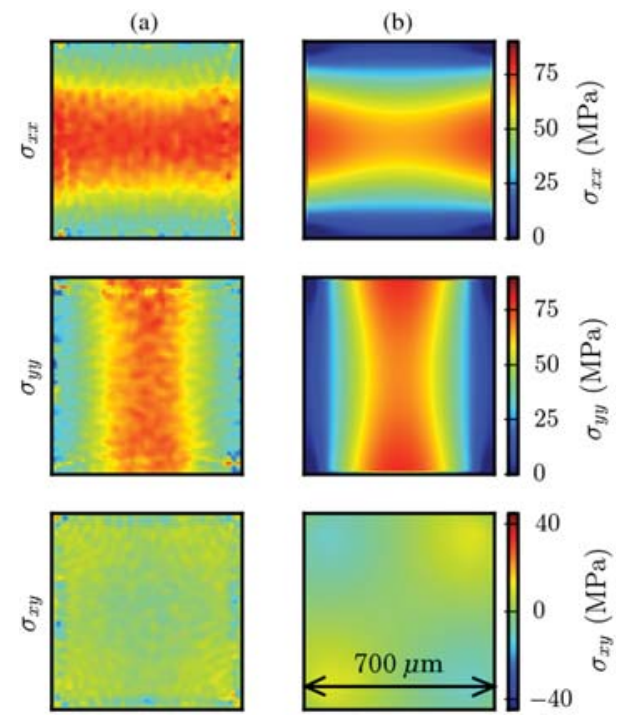

FIG. 4. False color images of the components $\sigma_{i j}$ of the stress tensor in a silicon membrane bent by a pressure difference of 50 mbar. On the left hand side (a), the experimental data are shown, obtained using the method presented in this paper. For comparison, the results of finite element (FE) simulations are shown on the right hand side (b).

components $\sigma_{x x}$ and $\sigma_{y y}$, there is good quantitative agreement. For the shear stress $\sigma_{x y}$, there is agreement in the order of magnitude, but since it is much smaller than the diagonal components, the SNR is insufficient for quantitative comparison. For all the components, there are artifacts along the edges of the membrane, because the computation of the numerical derivatives fails there.

In Fig. 5, a false-color image of the stress of a siliconcarbide membrane is shown, with a cross-shaped area of reduced thickness and increased stress. The etch pattern is designed to produce sharp, clearly visible features for the stress pattern. Therefore, the sample is used to estimate the spatial resolution of the method presented in this paper. To produce this image, 24 modes in the frequency range from 0.4 to $1.7 \mathrm{MHz}$ are analyzed. For comparison, we perform finite-element simulations. The geometry of the sample from the experiment is captured by optical microscopy, ellipsometry, and atomic-force microscopy in order to design an accurate model for the simulation. The prestress in the membrane plane is homogeneous and isotropic but we do not know much about the stress distribution in the normal direction. The simplest model that fits the experimental results is a linear stress gradient with a tensile stress of $200 \mathrm{MPa}$ at the bottom and zero at the top side of the membrane. The stress-tensor components from a simulation using this model are shown on the right-hand side of Fig. 5. We know that the average stress is $78 \mathrm{MPa}$, from measurements of the dispersion relation of bending waves of unpatterned silicon-carbide membranes fabricated from the same wafer. To estimate the stress gradient, we cut a
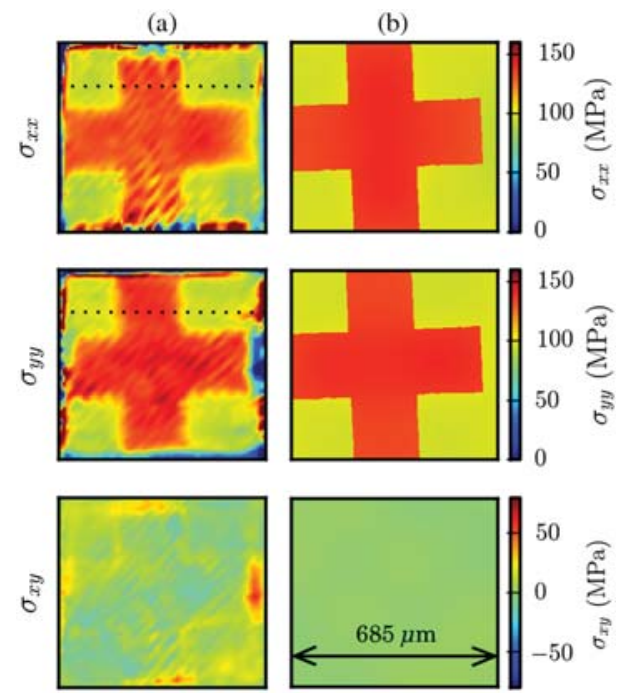

FIG. 5. False color images of the components $\sigma_{i j}$ of the stress tensor in a prestressed silicon carbide membrane. The thickness is $264 \mathrm{~nm}$ outside and $169 \mathrm{~nm}$ inside of the cross shaped area. The experimental results (a) are compared to the results of finite element simulations (b). The sharp features of the stress pattern are used to estimate the spatial resolution of $30 \mu \mathrm{m}$, which is much better than the smallest wavelength $\lambda>115 \mu \mathrm{m}$ of the measured modes.

membrane flake out of the frame. From the curling radius $R$ of the now free membrane, the stress gradient is calculated [22] $\partial \sigma / \partial z=E /\left(1-\nu^{2}\right) R$. That way, we obtain a stress difference of approximately $150 \mathrm{MPa}$ from the top to the bottom side. Both values are about $25 \%$ lower than the values used for the simulation, which is a good agreement, given the rough approximation of a linear stress distribution.

In order to estimate the spatial resolution, we compare the experimental data on the left-hand side of Fig. 5 with the finite-element simulations shown on the right-hand side. At the edges and corners of the cross pattern, the stress from the simulation is discontinuous on the micron scale.

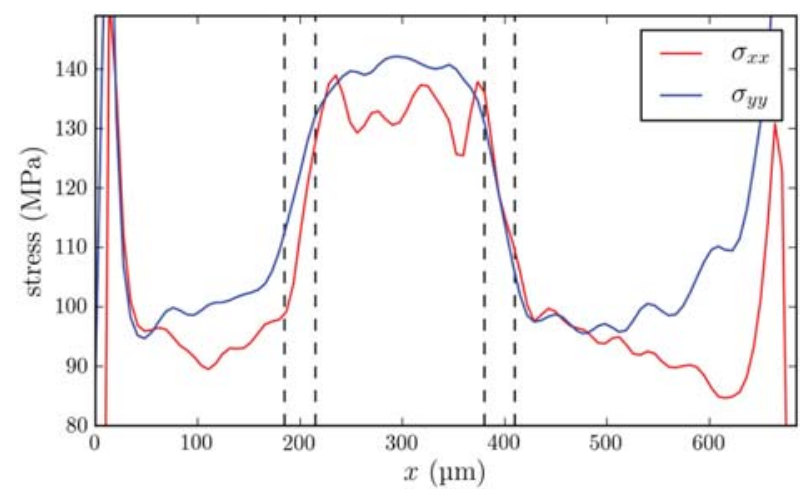

FIG. 6. Cross sections through the panels for $\sigma_{x x}$ and $\sigma_{y y}$ of Fig. 5 taken at the dotted lines. The vertical dashed lines indicate the uncertainty of the determination of the edges of the stress pattern. 
The experimental data show a smooth transition with a width of $30 \mu \mathrm{m}$, as visualized by the vertical dashed lines in Fig. 6. Therefore, spatial resolution is much better than the smallest wavelength, $\lambda>115 \mu \mathrm{m}$, of the measured modes used to compute the experimental values for the stress tensor. This comparison proves our claim that the wavelength limit for the resolution does not apply to this method. The resolution is limited by the Gaussian bandpass filter applied to $z$ and by the diffraction limit of the optical profilometer of 2-3 $\mu \mathrm{m}$. While it is possible to measure wavelengths down to $23 \mu \mathrm{m}$ with our setup (based on the necessity to capture roughly 10 pixels per wavelength to map the wave shape with sufficient precision), the signal-to-noise ratio decreases drastically for high frequencies. The minimum wavelength used here is given by the mode-selection filter. By adjusting the parameters of the Gaussian bandpass filter and the mode selection filter, we find a compromise between spatial resolution and good signal-to-noise ratio in the stress maps.

\section{POSSIBLE APPLICATIONS TO OTHER FIELDS OF PHYSICS}

In principle, the method presented above is applicable to a large variety of systems in physics. The only requirements are an equation that can be written in the form of Eq. (4), as well as the complete knowledge of the function $z$ for several modes or configurations. Of course, the necessity to measure the complete state of the system $z$ is an important limitation for practical purposes. On the other hand, there are very few preconditions on the structure of the equation:

(a) In our case, the equation used is the equation of motion, but this is not a requirement.

(b) In our example, $z:(\vec{r}, t) \rightarrow \mathbb{C}$ is scalar. In general, it can be a vector function $z: \mathbb{R}^{d} \rightarrow \mathbb{C}^{n}$.

(c) The equation must be linear in $p_{l}$, but it may be nonlinear in $z$, since the operators $\hat{X}_{l}$ are allowed to be nonlinear.

(d) Equation (4) seems to be homogeneous, but an inhomogeneity $f(\vec{r}, t)$ can be added by adding an additional addend $\hat{X}_{0} z=f(\vec{r}, t)$, with the nonlinear operator $\hat{X}_{0}$, which does not depend on the field $z(\vec{r})$. In many cases, the parameters $p_{l}$ in Eq. (4) are not independent, but some are partial derivatives of others. As an example, we will discuss diffusion in inhomogeneous systems. On the one hand, methods to measure the concentration [23] or the temperature [24] in particle or heatdiffusion systems as a function of space and time are known. On the other hand, inhomogeneous diffusion is a topic of continuing theoretical interest, which has been discussed for several decades [25-31]. In order to know the diffusion law, the microscopic nature of the system has to be examined [27]. We will use the simplest one, Fick's law in two dimensions,

$$
0=-\frac{\partial n}{\partial t}+\mathcal{D} \Delta n+\frac{\partial \mathcal{D}}{\partial x} \frac{\partial n}{\partial x}+\frac{\partial \mathcal{D}}{\partial y} \frac{\partial n}{\partial y},
$$

with the concentration $n$ and the diffusion constant $\mathcal{D}$. For our method, there are three free-fit parameters, $\mathcal{D}$ and its two spatial derivatives. If the experimental uncertainty for the measurement of $n$ is low enough, a fit with three parameters might give good results. In this case, the experimentally obtained derivatives of $\mathcal{D}$ could be compared to numerical ones, to verify the diffusion equation that is used.

If the diffusion constant varies only slowly compared to the length scale of concentration changes, the derivatives of $\mathcal{D}$ can be neglected. In this case, there is only one free parameter left, but the spatial resolution of the method is limited by this length scale.

\section{CONCLUSION AND SUMMARY}

We study bending waves in silicon and silicon-carbide membranes with inhomogeneous anisotropic prestress. We present an algorithm to calculate the lateral components of the stress tensor as a function of location from the experimentally observed mode shapes at several frequencies. The spatial resolution achieved is much better than the wavelengths of the modes. The results show nice agreement with finite-element simulations. In principle, the method is applicable to a wide range of systems, in order to measure inhomogeneities in material parameters, not only in mechanics. The main limitation is the necessity to measure the state of the system as a function of space and time.

\section{ACKNOWLEDGMENTS}

This work was funded by the DFG through Grant No. SFB767 and the Excellence Initiative of the German Federal Government and the State of Baden-Württemberg through Grant No. ZUK52/2. We thank Ph. Tanner for providing us with silicon-carbide-coated wafers and $\mathrm{P}$. Reimann, X. Zhang, P. Leiderer, T. Dekorsy, and the members of the Nanomechanics Discussion Group of SFB767 for valuable discussions. Technical, experimental, and programming contributions of A. Fischer, S. Diesch, and F. Yang are gratefully acknowledged.

[1] J. J. Milsom and A. Eriksen, Field Geophysics, 4th ed. (Wiley, New York, 2011).

[2] R. J. Urick, Principles of Underwater Sound, 3rd ed. (McGraw Hill, New York, 1983).

[3] H. Kuttruff, Physik und Technik des Ultraschalls (S. Hirzel Verlag, Stuttgart, 1988).

[4] K. J. Langenberg, R. Marklein, and K. Mayer, Ultrasonic Nondestructive Testing of Materials (CRC Press, Boca Raton, FL, 2012).

[5] N.W. Ashcroft and D. N. Mermin, Festkörperphysik, 2nd ed. (Oldenbourg Wissenschaftsverlag, Munich, 2005).

[6] C. Thomsen, J. Strait, Z. Vardeny, H. J. Maris, J. Tauc, and J. J. Hauser, Coherent Phonon Generation and Detection by Picosecond Light Pulses, Phys. Rev. Lett. 53, 989 (1984). 
[7] A. Bruchhausen, R. Gebs, F. Hudert, D. Issenmann, G. Klatt, A. Bartels, O. Schecker, R. Waitz, A. Erbe, E. Scheer, J. R. Huntzinger, A. Mlayah, and T. Dekorsy, Subharmonic Resonant Optical Excitation of Confined Acoustic Modes in a Free Standing Semiconductor Membrane at $\mathrm{GHz}$ Frequencies with a High Repetition Rate Femtosecond Laser, Phys. Rev. Lett. 106, 077401 (2011).

[8] R. Waitz, S. Nößner, M. Hertkorn, O. Schecker, and E. Scheer, Mode shape and dispersion relation of bending waves in thin silicon membranes, Phys. Rev. B 85, 035324 (2012).

[9] D. T. Cassidy, S. K. K. Lam, B. Lakshmi, and D. M. Bruce, Using local band structure to image strain in semiconductor microstructures, Appl. Opt. 43, 1811 (2004).

[10] T. H. Stievater, W. S. Rabinovich, D. Park, P. G. Goetz, J. B. Boos, D. S. Katzer, and M. L. Biermann, Using local band structure to image strain in semiconductor microstructures, Appl. Phys. Lett. 86, 111915 (2005).

[11] J. W. Tomm, T. Q. Tien, and D. T. Cassidy, Spectroscopic strain measurement methodology: Degree of polarization photoluminescence versus photocurrent spectroscopy, Appl. Phys. Lett. 88, 133504 (2006).

[12] J. Butschke, A. Ehrmann, E. Haugeneder, M. Irmscher, R. Kaesmaier, K. Kragler, F. Letzkus, H. Loeschner, J. Mathuni, I. W. Rangelow, C. Reuter, F. Shi, and R. Springer, $\mathrm{PN}$ and SOI wafer flow process for stencil mask fabrication, Proc. SPIE Int. Soc. Opt. Eng. 3665, 20 (1999).

[13] L. Wang, S. Dimitrijev, J. Han, A. Iacopi, L. Hold, P. Tanner, and H. B. Harrison, Growth of $3 \mathrm{C} \mathrm{SiC}$ on $150 \mathrm{~mm} \mathrm{Si}(100)$ substrates by alternating supply epitaxy at $1000^{\circ} \mathrm{C}$, Thin Solid Films 519, 6443 (2011).

[14] V. Ziebart, O. Paul, and H. Baltes, Strongly buckled square micromachined membranes, J. Microelectromech. Syst. 8, 423 (1999).

[15] P. Murray and G. F. Carey, Determination of interfacial stress during thermal oxidation of silicon, J. Appl. Phys. 65, 3667 (1989).

[16] A. N. Cleland, Foundations of Nanomechanics: From Solid State Theory to Device Applications (Springer Verlag, Berlin, 2003).
[17] L. D. Landau and E. M. Lifshitz, Lehrbuch der Theoretischen Physik, Band VII Elastizitätstheorie (Akademie Verlag, Berlin, 1991).

[18] K.F. Graff, Wave Motion in Elastic Solids (Oxford University Press, London, 1975).

[19] E. V. Nolde, L. A. Prikazchikova, and G. A. Rogerson, Dispersion of small amplitude waves in a pre stressed, compressible elastic plate, J. Elast. 75, 1 (2004).

[20] Webelements: The periodic table on the web, http://www .webelements.com/silicon/.

[21] G. L. Harris, Properties of Silicon Carbide, Vol. 13 (INSPEC, London, 1995).

[22] S. Timoshenko and S. Woinowsky Krieger, Theory of Plates and Shells (McGraw Hill, New York, 1959), Vol. 2.

[23] J. Capoulade, M. Wachsmuth, L. Hufnagel, and M. Knop, Quantitative fluorescence imaging of protein diffusion and interaction in living cells, Nat. Biotechnol. 29, 835 (2011).

[24] M. Schmotz, P. Bookjans, E. Scheer, and P. Leiderer, Optical temperature measurements on thin freestanding silicon membranes, Rev. Sci. Instrum. 81, 114903 (2010).

[25] R. Stratton, Diffusion of hot and cold electrons in semi conductor barriers, Phys. Rev. 126, 2002 (1962).

[26] N. G. van Kampen, Diffusion in inhomogeneous media, J. Phys. Chem. Solids 49, 673 (1988).

[27] B. Ph. van Milligen, P. D. Bons, B. A. Carreras, and R. Sánchez, On the applicability of Fick's law to diffusion in inhomogeneous systems, Eur. J. Phys. 26, 913 (2005)

[28] F. Sattin, Fick's law and Fokker Planck equation in inhomogeneous environments, Phys. Lett. A 372, 3941 (2008).

[29] E. Bringuier, Kinetic theory of inhomogeneous diffusion, Physica (Amsterdam) 388A, 2588 (2009).

[30] E. Cho and Y. J. Kim, Starvation driven diffusion as a survival strategy of biological organisms, Bull. Math. Biol. 75, 845 (2013).

[31] J. Alvarez Ramirez, L. Dagdug, and M. Meraz, Asymmetric diffusion in heterogeneous media, Physica (Amsterdam) 395A, 193 (2014). 